\title{
SMOOTH EXTENSIONS FOR FINITE CW COMPLEXES
}

\author{
GUIHUA GONG
}

ABSTRACT. In this paper, we have completely classified the $C_{n}$-smooth elements of $\operatorname{Ext}(X)$ modulo torsion for $X$ being an arbitrary finite $\mathrm{CW}$ complex.

\section{INTRODUCTION}

Let $H$ be an infinite dimensional complex separable Hilbert space. By $L(H)$ and $K(H)$ we shall denote the $C^{*}$-algebras of bounded operators and compact operators respectively on $H$, and $Q(H)$ will denote the quotient $L(H) / K(H)$ with canonical surjection $\pi: L(H) \rightarrow Q(H)$.

Let $X$ be a compact metrizable space. By $C(X)$ we shall denote the $C^{*}$ algebra of continuous complex valued functions on $X$. An extension $\tau \in$ $\operatorname{Ext}(X)$ of the algebra $C(X)$ by $K(H)$ is defined by a unital $*$ monomorphism $\tau: C(X) \rightarrow Q(H)[6]$.

For two extensions $\tau_{1}$ and $\tau_{2}$ of $C(X)$ by $K(H)$, we say $\tau_{1}$ and $\tau_{2}$ are equivalent if there exists a unitary operator $U$ on $H$ such that $\tau_{1}(f)=$ $\pi\left(U^{*}\right) \tau_{2}(f) \pi(U)$. By $\operatorname{Ext}(X)$ we shall denote the collection of equivalence classes of extensions of $C(X)$ by $K(H)$.

Let $\tau_{1}$ and $\tau_{2}$ be $*$ monomorphisms from $C(X)$ into $Q(H)$ and $a_{1}=\left[\tau_{1}\right]$ and $a_{2}=\left[\tau_{2}\right]$ denote the elements of $\operatorname{Ext}(X)$ they determine. Further, let $\rho: Q(H) \oplus Q(H) \rightarrow Q(H)$, be the map determined by the diagram

$$
\begin{array}{cccc}
L(H) \oplus L(H) & \rightarrow L(H \oplus H) \stackrel{\nu}{\rightarrow} & L(H) \\
\downarrow \pi \oplus \pi & & & \downarrow \pi \\
Q(H) \oplus Q(H) & \stackrel{\rho}{\rightarrow} & & Q(H)
\end{array}
$$

where $\nu$ is induced by any unitary between $H \oplus H$ and $H$. Now if $\tau: C(X) \rightarrow$ $Q(H)$ is the map defined by

$$
\tau(f)=\rho\left(\tau_{1}(f) \oplus \tau_{2}(f)\right)
$$

for $f$ in $C(X)$, then we set $a_{1}+a_{2}=[\tau]$. One can verify that $[\tau]$ does not depend on the choice of $\nu$.

An extension $\tau: C(X) \rightarrow Q(H)$ is said to be trivial if there exists a unital * monomorphism $\sigma: C(X) \rightarrow L(H)$ such that $\tau=\pi \circ \sigma$. The basic fact of $C^{*}$ -

Received by the editors January 29, 1991 and, in revised form, February 7, 1992.

1991 Mathematics Subject Classification. Primary 46L80; Secondary 19K33, 46M20.

This paper is one part of the author's thesis completed in SUNY at Stony Brook under the supervision of Professor Ronald G. Douglas. The author was partially supported by NSF as a graduate assistant. 
algebra extension theory is that $\operatorname{Ext}(X)$ is an abelian group with the equivalence class of the trivial extension as the unit.

We refer to [10 and 4] for the basic theory of $C^{*}$-algebra extensions.

$\operatorname{Ext}(X)$ was introduced by Brown, Douglas and Fillmore in order to classify essentially normal operators up to unitary equivalence modulo the compact operators. It can be proved that $\operatorname{Ext}(X)$ is isomorphic to $K$-homology $K_{1}(X)$ (defined by using Spanier-Whitehead duality). But $\operatorname{Ext}(X)$ has a close relation with the index theory of elliptic operators $[1,10]$ and is a starting point of $K K$ theory. Furthermore, this kind of functor can be defined for "noncommutative spaces" which is useful to give a new invariant for group actions and foliations [9].

Let $G l_{n}(\mathbb{C})$ and $U(n)$ be the topological groups of $n \times n$ complex invertible matrices and $n \times n$ unitary matrices respectively. $K^{1}(X)$ is defined to be the collection of homotopy equivalence classes of maps from $X$ to $\bigcup_{n=1}^{\infty} G l_{n}(\mathbb{C})$ or maps from $X$ to $\bigcup_{n=1}^{\infty} U(n)$ (note $G l_{n}(\mathbb{C})$ and $U(n)$ are homotopy equivalent). Therefore an element in $K^{1}(X)$ can be represented by a map $\theta: X \rightarrow$ $G l_{n}(\mathbb{C})$ or $\theta: X \rightarrow U(n)$ for $n$ large enough.

Now we will establish the pairing between $\operatorname{Ext}(X)$ and $K^{1}(X)$. Let $\tau \in$ $\operatorname{Ext}(X)$ and $\theta \in K^{1}(X)$. First we note that $\theta: X \rightarrow G l_{n}(C)$ can be regarded as an invertible element in $C(X) \otimes M_{n}$. Therefore $\left(\tau \otimes 1_{n}\right)(\theta)$ is an invertible element in

$$
Q(\underbrace{H \oplus H \oplus \cdots \oplus H}_{n \text {-copies }}) .
$$

The pairing of $\tau$ and $\theta$ is defined by

$$
\langle\tau, \theta\rangle=\operatorname{index}\left(\left(\tau \otimes 1_{n}\right)(\theta)\right) \in \mathbb{Z}
$$

Before studying $\operatorname{Ext}(X)$ and $K^{1}(X)$, some topological results will be proved in $\S 1$ which will be used in later sections and are interesting in their own right. In particular, we prove the following useful theorem which enables us to reduce some problems from the general case to the case of spheres.

Theorem 1.2. Let $X$ be a compact metrizable space. For any $\tau \in K^{1}(X)$, there exist maps $f_{i}: X \rightarrow S^{2 n_{i}-1} \quad(i=1,2, \ldots, k)$ such that $m \tau=\sum_{i=1}^{k} f_{i}^{*} \theta_{i}$ for some integer $m \neq 0$ and $\theta_{i} \in K^{1}\left(S^{2 n_{i}-1}\right)$.

In $\S 2$, we study the $C_{p}$-smooth extensions of finite $\mathrm{CW}$ complexes and finite $\mathrm{CW}$ complex pairs. The following definition of $C_{p}$-smoothness for smooth manifolds can be found in [8].

Definition 0.1. Let $M$ be a smooth compact manifold (perhaps with boundary) and let $C^{\infty}(M)$ denote the *-algebra of all smooth functions on $M$. A $\tau \in$ $\operatorname{Ext}(M)$ is $C_{p}$-smooth if there exists a $*$-linear map $\rho: C^{\infty}(M) \rightarrow L(H)$ such that $\rho(a b)-\rho(a) \rho(b) \in C_{p}$ and $\pi \circ \rho=\left.\tau\right|_{C^{\infty}(M)}$.

The notion of $C_{p}$-smoothness was introduced by R. Douglas and is one source of the motivation for Connes noncommutative geometry.

It was shown in $[17,11]$ that the $C_{1}$-smooth elements of $\operatorname{Ext}(X)$ come from the 1-skeleton of $X$ modulo torsion. And also it was shown in [12] that each $C_{n-1}$-smooth element of $\operatorname{Ext}\left(S^{2 n-1}\right)$ is trivial. The natural problem is to classify $C_{p}$-smooth extensions modulo torsion for a general $\mathrm{CW}$ complex. 
In $\S 2$, we generalize Definition 0.1 to a finite $\mathrm{CW}$ complex and obtain the following theorem which solves the above problem. Especially, the results in $[17,11$ and 12] are direct consequences of our theorem.

Theorem $2.2 \&$ Theorem 2.4. Let $X$ be a finite $C W$ complex, $X^{k}$ denote the $k$-skeleton of $X$, and $\tau \in \operatorname{Ext}(X)$. Then there exists an integer $m_{1} \neq 0$ such that $m_{1} \tau$ is $C_{n}$-smooth if and only if there exists an integer $m_{2} \neq 0$ such that $m_{2} \tau \in i_{*}\left(\operatorname{Ext}\left(X^{2 n-1}\right)\right)$, where $i_{*}: \operatorname{Ext}\left(X^{2 n-1}\right) \rightarrow \operatorname{Ext}(X)$ is induced by the inclusion map $i: X^{2 n-1} \rightarrow X$. Furthermore, if $X$ is a smooth compact $(2 n-1)$ manifold, then each element in $\operatorname{Ext}(X)$ is $C_{p}$-smooth when $p>n-\frac{1}{2}$.

Theorem 2.3. Let $X$ be a finite $C W$ complex, $\tau \in \operatorname{Ext}(X)=K_{1}(X)$ and ch: $K_{1}(X) \otimes \mathbb{Q} \rightarrow H_{\text {odd }}(X, \mathbb{Q})$ be the Chern map, where $H_{\text {odd }}(X, \mathbb{Q})$ denotes the direct sum of all the ordinary homology groups of odd dimension with rational coefficients. Then there exists an integer $m \neq 0$ such that $m \tau$ is $C_{n}$-smooth if and only if $\operatorname{ch} \tau \in \bigoplus_{k=1}^{n} H_{2 k-1}(X, \mathbb{Q})$.

The main idea in the proofs of our theorems is using some topological techniques (e.g., Theorem 1.2) to reduce the case of $\mathrm{CW}$ complexes to the case of spheres (for the "only if" part) which studied in [12], or to the case of compact smooth manifolds (for the "if" part).

More generally, we also obtain similar results for the relative extension theory of finite $\mathrm{CW}$ complex pairs.

In $\S 3$, we study $p$-summable Fredholm modules of $C^{\infty}(M)$, which can be thought of as elements of $K_{0}(M)=K K(C(M), \mathbb{C})$, and their Chern characters in the cyclic cohomology $H_{\lambda}^{*}\left(C^{\infty}(M)\right)$, where $H_{\lambda}^{*}\left(C^{\infty}(M)\right)$ is an analogue of deRham homology theory obtained by first using algebra language which then can be generalized to noncommutative algebras. (See [8] for details.) We will say more about $H_{\lambda}^{*}\left(C^{\infty}(M)\right)$ in $\S 3$. In particular, we prove the following theorem.

Theorem 3.2. If $M$ is a compact smooth manifold without boundary and $\varphi \in$ $H_{\lambda}^{k}\left(C^{\infty}(M)\right)$ ( $k$ even), then there exist $(k+1)$-summable Fredholm modules $\tau_{i}(i=1,2, \ldots, n)$ and complex numbers $\alpha_{i}(i=1,2, \ldots, n)$ such that $\sum_{i=1}^{n} \alpha_{i} \mathrm{ch}^{*} \tau_{i} \sim \varphi$ in $H_{\lambda}^{*}\left(C^{\infty}(M)\right)$, where $\mathrm{ch}^{*}$ is Connes' Chern map.

We would like to point out that $\mathrm{A}$. Connes constructed the graded Chern characters

$$
\mathrm{ch}^{*}:\{(n+1) \text {-summable Fredholm modules }\} \rightarrow H_{\lambda}^{n}\left(C^{\infty}(M)\right)
$$

in $\S 2$ of [8], where $n$ is an even integer, and that he also proved that

$$
\mathrm{ch}^{*}:\{\text { finite summable Fredholm modules }\} \rightarrow H^{\text {even }}\left(C^{\infty}(M)\right)
$$

is surjective modulo torsion. Theorem 3.2 says that the Chern map is a graded surjection.

Some results of the paper have been announced in [13].

\section{SOME TOPOLOGICAL RESUltS}

In this section, we will prove some topological results. Theorem 1.1 will be used in defining $C_{p}$-smooth extension for general finite $\mathrm{CW}$ complexes. Theorem 1.2 will be used in proving our main results in $\S 2$ and [14]. Theorem 1.3 will be used in $\S \S 2$ and 3. 
Theorem 1.1. If $X$ is a finite $C W$ complex, then there exists a compact smooth manifold $M$ (perhaps with boundary), and two maps $f: X \rightarrow M$ and $g: M \rightarrow$ $X$ such that $g \circ f$ is homotopic to $\left.\mathrm{id}\right|_{X}$.

Using the following proposition [15], we can reduce the proof of this theorem to the case of $X$ being a simplicial complex.

Proposition 1.1. Every $C W$ complex has the homotopy type of a simplicial complex.

Before proving Theorem 1.1, we prove the following lemma.

Lemma 1.1. For any finite simplicial complex $X$, there exists an embedding $i: X \rightarrow R^{n}$ (for some $n$ ) and an open neighborhood $U$ of $i(X)$ such that $i(X)$ is a retract of $U$.

Proof. Let $\left\{v_{1}, v_{2}, \ldots, v_{n}\right\}$ be the vertices of $X$. We can define $i: X \rightarrow R^{n}$ to be the piecewise linear map determined by

$$
i\left(v_{k}\right)=(\underbrace{0, \ldots, 0}_{k-1}, 1, \underbrace{0, \ldots, 0}_{n-k}) \in R^{n} .
$$

Let $Y=\left\{\left(a_{1}, a_{2}, \ldots, a_{n}\right) \in R^{n} \mid-2 \leq a_{k} \leq 2\right.$ for all $\left.k\right\}$. It is easy to see that $Y$ is a closed neighborhood of $i(X)$ in $R^{n}$. We can triangulate $Y$ so that $i(X)$ is a subcomplex of $Y$. The result of (1.6) on p. 50 of [22] says that $(Y, i(X))$ is an NDR pair. This means that there is an open neighborhood $U_{1}$ of $X$ in $Y$ such that $X$ is a retract of $U_{1}$. If $U_{1}$ is not open in $R^{n}$, we can replace $U_{1}$ by a smaller open neighborhood $U$ of $X$. Q.E.D.

Proof of Theorem 1.1. By Lemma 1.1, without loss of generality, we can assume $X$ is a closed subset of $R^{n}$ with an open neighborhood $U$ such that $X$ is a retract of $U$.

Define a map $u: R^{n} \rightarrow R$ by $u(x)=d(x, X)$ (for all $x \in R^{n}$ ) which is the distance between the point $x$ and the closed subset $X$. Then there exists an $\varepsilon>0$ such that $\{x \mid u(x)<3 \varepsilon\} \subset U$.

It is well known that there exists a smooth map $v: R^{n} \rightarrow R$ (see Proposition 17.8 on p. 213 of [5]) with $|u(x)-v(x)|<\varepsilon$ for all $x \in R^{n}$. By the Sard Theorem, there exists a regular value $c$ of the map $v$ in $(\varepsilon, 2 \varepsilon)$. So if we choose $M=\{x \mid v(x) \leq c\}$, then $M$ is a compact smooth manifold with smooth boundary $\{x \mid v(x)=c\}$. It is easy to see that $X \subset M \subset U$. So $X$ is a retract of $M$. We can define the map $f$ to be the inclusion from $X$ to $M$, and the map $g$ to be a retraction from $M$ to $X$, thus completing the proof of Theorem 1.1. Q.E.D.

We believe that Theorem 1.1 is a well-known result in topology. We provide a proof here because we have been unable to find a precise reference for it.

Our next aim is to prove the following main theorem in this chapter.

Theorem 1.2. Let $X$ be a compact metrizable space. For any $\tau \in K^{1}(X)$, there exist maps $f_{i}: X \rightarrow S^{2 n_{i}-1} \quad(i=1,2, \ldots, k)$ such that $m \tau=\sum_{i=1}^{k} f_{i}^{*} \theta_{i}$ for some integer $m \neq 0$ and $\theta_{i} \in K^{1}\left(S^{2 n_{i}-1}\right)$.

The proof of Theorem 1.2 will be divided into several steps. 
Lemma 1.2. If Theorem 1.2 is true for the special case of $X=U(n)$, then the theorem is true for an arbitrary compact metrizable space $X$.

Proof. Assume the theorem is true for $U(n)$ and $X$ is a compact metrizable space. Let $\tau \in K^{1}(X)$. Then $\tau$ can be realized as a map $f: X \rightarrow U(n)$ for $n$ large enough. Let $\hat{\tau} \in K^{1}(U(n))$ be the element determined by the identity map from $U(n)$ to $U(n)$. Hence $\tau=f^{*} \hat{\tau}$. By our assumption $m \hat{\tau}=\sum_{i=1}^{k} f_{i}^{*} \theta_{i}$ for some maps $f_{i}: U(n) \rightarrow S^{2 n_{i}-1}, \theta_{i} \in K^{1}\left(S^{2 n_{i}-1}\right)$ and integer $m \neq 0$. Therefore $m \tau=\sum_{i=1}^{k}\left(f \circ f_{i}\right)^{*} \theta_{i}$. This completes the proof of Lemma 1.2. Q.E.D.

Lemma 1.3. Theorem 1.2 is true for $X=S^{1} \times S^{3} \times \cdots \times S^{2 n-1}$.

Proof. Consider ordinary cohomology $H^{*}(X)=\bigoplus_{i} H^{i}(X)$. The Künneth formula yields

$$
H^{*}(X)=H^{*}\left(S^{1}\right) \otimes H^{*}\left(S^{3}\right) \otimes H^{*}\left(S^{5}\right) \otimes \cdots \otimes H^{*}\left(S^{2 n-1}\right) .
$$

Let $\tau_{1}, \tau_{3}, \ldots, \tau_{2 n-1}$ be generators of $H^{1}\left(S^{1}\right), H^{3}\left(S^{3}\right), \ldots, H^{2 n-1}\left(S^{2 n-1}\right)$, respectively. Then $H^{*}(X)$ is generated by $1, \tau_{1}, \tau_{3}, \ldots, \tau_{2 n-1}, \tau_{1} \times \tau_{3}, \tau_{1} \times$ $\tau_{5}, \ldots, \tau_{1} \times \tau_{3} \times \tau_{5} \times \cdots \times \tau_{2 n-1}$ as a group. But each $\tau_{p_{1}} \times \tau_{p_{2}} \times \cdots \times \tau_{p_{k}}$ $\left(1 \leq p_{i} \leq 2 n-1\right.$ are odd numbers) is a generator of

$$
H^{p_{1}+p_{2}+\cdots+p_{k}}\left(S^{p_{1}} \times S^{p_{2}} \times \cdots \times S^{p_{k}}\right) .
$$

Let $f$ be the canonical map of degree 1 from $S^{p_{1}} \times S^{p_{2}} \times \cdots \times S^{p_{k}}$ to $S^{p_{1}+p_{2}+\cdots+p_{k}}$ (this map collapses $\bigcup_{i=1}^{k} S^{p_{1}} \times S^{p_{2}} \times \cdots \times S^{p_{i-1}} \times\{\mathrm{pt}\} \times S^{p_{i+1}} \times \cdots \times S^{p_{k}}$ to one point, where $\{\mathrm{pt}\}$ denotes a fixed point in $S^{p_{i}}$ ). Then $f$ induces an isomorphism from $H^{p_{1}+p_{2}+\cdots+p_{k}}\left(S^{p_{1}+p_{2}+\cdots+p_{k}}\right)$ to $H^{p_{1}+p_{2}+\cdots+p_{k}}\left(S^{p_{1}} \times S^{p_{2}} \times \cdots \times S^{p_{k}}\right)$. This proves $\tau_{p_{1}} \times \tau_{p_{2}} \times \cdots \times \tau_{p_{n}}=f^{*} \theta$ for some $\theta \in H^{p_{1}+p_{2}+\cdots+p_{k}}\left(S^{p_{1}+p_{2}+\cdots+p_{k}}\right)$. Therefore, for any element $\tau \in H^{*}(X)\left(X=S^{1} \times S^{3} \times \cdots \times S^{2 n-1}\right)$ there exist maps $f_{i}: X \rightarrow S^{n_{i}}$ such that $\tau=\sum f_{i}^{*} \theta_{i}$ for some $\theta_{i} \in H^{*}\left(S^{n_{i}}\right)$.

By using bijectivity (up to a rational multiplier) and the naturality of the Chern map between $K^{*}(X)$ and $H^{*}(X)$, one verifies Lemma 1.3 easily. Q.E.D.

By Lemma 1.2 and Lemma 1.3, we need only to reduce the case of $X=U(n)$ to the case of $X=S^{1} \times S^{3} \times \cdots \times S^{2 n-1}$ for proving Theorem 1.2.

First, we recall some results on the topology of $U(n)$. Let $p_{n}: U(n) \rightarrow S^{2 n-1}$ be the map which maps $A \in U(n)$ to the last column of $A$ which can be thought of as an element in $S^{2 n-1}$. Then we have the fibre bundle

$$
U(n-1) \stackrel{i_{n}}{\rightarrow} U(n) \stackrel{p_{n}}{\rightarrow} S^{2 n-1},
$$

where $i_{n}$ is the inclusion map which maps $B \in U(n-1)$ to $\left(\begin{array}{ll}B & 0 \\ 0 & 1\end{array}\right) \in U(n)$.

Suppose $m<n$, and consider the finite CW complex pair $(U(n), U(m))$, where $U(m)$ is embedded in $U(n)$ by the inclusion map which maps $B \in U(m)$ to

$$
\left(\begin{array}{cc}
B & 0 \\
0 & 1_{n-m}
\end{array}\right) \in U(n)
$$

The following lemma is about the cell structure of the pair $(U(n), U(m))$.

Lemma 1.4. There exists a finite $C W$ complex $X$ with $U(m)$ as the $2 m$-skeleton of $X$ and a homotopy equivalence $f: U(n) \rightarrow X$ such that $\left.f\right|_{U(m)}=\mathrm{id}$.

Proof. We need only to prove the case $n=m+1$. Consider the fibre bundle $U(m) \rightarrow U(m+1) \rightarrow S^{2 m+1}$. 
By Theorem 8.5 on p. 187 of [22], we have $\pi_{k}(U(m+1), U(m))=\pi_{k}\left(S^{2 m+1}\right)$ for all $k \geq 1$. Hence $\pi_{k}(U(m+1), U(m))=0$ whenever $0 \leq k \leq 2 m$.

Theorem 2.6 on p. 219 of [22] implies that any $n$-connected CW pair $(X, A)$ is weakly homotopy equivalent to a $\mathrm{CW}$ pair $(Y, A)$ with $A$ being the $n$ skeleton of $Y$. And Theorem 3.5 on p. 220 of [22] says that weakly homotopy equivalence between two $\mathrm{CW}$ pairs is homotopy equivalence. Put these two theorems together to finish the proof of Lemma 1.4. Q.E.D.

As in the proof of Lemma 1.4, if $(X, A)$ is an $n$-connected $\mathrm{CW}$ pair, we can always assume that $A$ is the $n$-skeleton of $X$. In particular, if $X$ is an $n$-connected CW complex, we can assume that the $n$-skeleton of $X$ is a set consisting of a single point. This argument will be used several times in this paper.

Lemma 1.5. $H^{*}(U(n))=\mathbb{Z}\left\{x_{1}, x_{3}, \ldots, x_{2 n-1}\right\}$ is an exterior algebra with odd dimensional generators $\left\{x_{2 i-1}\right\}_{i=1}^{n}$. In addition, the following statements are true:

1. If $i: U(m) \rightarrow U(n) \quad(m<n)$ is the inclusion, then $i^{*}: H^{*}(U(n))=$ $\mathbb{Z}\left\{x_{1}, x_{3}, \ldots, x_{2 n-1}\right\} \rightarrow H^{*}(U(m))=\mathbb{Z}\left\{y_{1}, y_{3}, \ldots, y_{2 m-1}\right\}$ is defined by

$$
\begin{gathered}
i^{*} x_{2 i-1}=y_{2 i-1}, \quad \text { when } 0 \leq i \leq m, \\
i^{*} x_{2 i-1}=0, \quad \text { when } m+1 \leq i \leq n
\end{gathered}
$$

for a proper choice of generators $\left\{x_{2 i-1}\right\}_{i=1}^{n}$ and $\left\{y_{2 i-1}\right\}_{i=1}^{m}$.

2. If $p_{n}: U(n) \rightarrow S^{2 n-1}$ is defined as before, then $p_{n}^{*}$ maps the generator of $H^{2 n-1}\left(S^{2 n-1}\right)$ to $x_{2 n-1} \in H^{2 n-1}(U(n))$.

Proof. The first part of this lemma is a standard result in topology (see p. 164 in [16]) which can be proved by using the spectral sequence of the fibre bundle $U(n-1) \rightarrow U(n) \rightarrow S^{2 n-1}$. The additional parts (1) and (2) can be easily proved by using Lemma 1.4 and the above fibre bundle. Q.E.D.

Let $\chi_{k}^{(n)}$ denote a map of degree $k$ from $S^{2 n-1}$ to itself. Before proving the next lemma (which is a key lemma in proving Theorem 1.2), we state a result about homotopy operations.

Proposition 1.2. For any integer $k$ and any $[\alpha] \in \pi_{i}\left(S^{2 n-1}\right)$ represented by $\alpha: S^{i} \rightarrow S^{2 n-1}$ we have $\left[\chi_{4 k}^{(n)} \circ \alpha\right]=4 k[\alpha]$ in $\pi_{i}\left(S^{2 n-1}\right)$.

This proposition is a special case of Theorem 8.9 on p. 537 of [22] which is proved by using the Whitehead product.

Lemma 1.6. Let $(U(n), U(m))$ be as before $(m<n)$. Then there exists a $k \neq 0$ such that $\chi_{k}^{(m)} \circ p_{m}: U(m) \rightarrow S^{2 m-1}$ can be extended to a map from $U(n)$ to $S^{2 m-1}$.

Proof. We use $\chi_{k}, p$ to denote $\chi_{k}^{(m)}$ and $p_{m}$ respectively, for short. Let $X$ be the space in Lemma 1.4 , where we replace $U(n)$ by $X$. Let $X^{i}$ denote the $i$-skeleton of $X$. Then $X^{2 m}=U(m)$. We will prove by induction that there exists an integer $k_{i} \neq 0$ such that $\chi_{k_{i}} \circ p: U(m) \rightarrow S^{2 m-1}$ can be extended to $X^{i}$. Assume that there exists an integer $k_{i} \neq 0$ such that $\chi_{k_{i}} \circ p$ can be extended to $X^{i} \quad(i \geq 2 m)$. We are going to prove that there exists an integer $k_{i+1} \neq 0$ such that $\chi_{k_{i+1}} \circ p$ can be extended to $X^{i+1}$. Because $i \geq 2 m$, by 
Serre's Theorem, $\pi_{i}\left(S^{2 m-1}\right)$ is a finite group. Let $N$ be the order of this group. Choose $k_{i+1}=4 N k_{i}$. Then $\chi_{k_{i+1}} \circ p=\chi_{4 N} \circ\left(\chi_{k_{i}} \circ p\right)$ can be extended to $X^{i}$ (which we also denote by $\chi_{k_{i+1}} \circ p$ ) by the induction assumption.

Let $e^{i+1}$ be an arbitary $(i+1)$-cell of $X$ and $\theta: S^{i} \rightarrow \partial e^{i+1} \subset X^{i}$ be the attaching map of $e^{i+1}$. By obstruction theory, the map $\chi_{k_{i+1}} \circ p: X^{i} \rightarrow S^{2 m-1}$ can be extended to $e^{i+1}$ if and only if $\left(\chi_{k_{i+1}} \circ p\right) \circ \theta: S^{i} \rightarrow S^{2 m-1}$ defines a trivial map. But by Proposition 1.2

$$
\left[\left(\chi_{k_{i+1}} \circ p\right) \circ \theta\right]=\left[\chi_{4 N} \circ\left(\chi_{k_{i}} \circ p\right) \circ \theta\right]=4 N\left[\left(\chi_{k_{i}} \circ p\right) \circ \theta\right]=0
$$

in $\pi_{i}\left(S^{2 m-1}\right)$. This completes the proof of Lemma 1.6. Q.E.D.

Lemma 1.7. There exists a map $u: U(n) \rightarrow S^{1} \times S^{3} \times \cdots \times S^{2 n-1}$ such that for any $\tau \in H^{*}(U(n))$, there exists an integer $m \neq 0$ and $\theta \in H^{*}\left(S^{1} \times S^{3} \times \cdots \times S^{2 n-1}\right)$ with $m \tau=f^{*} \theta$.

Proof. By Lemma 1.6, we have maps $u_{i}: U(n) \rightarrow S^{2 i-1}(i=1,2, \ldots, n)$ which extend some $\chi_{k_{i}}^{(i)} \circ p: U(i) \rightarrow S^{2 i-1}$, where $k_{i} \neq 0(i=1,2, \ldots, n)$ are integers.

Let $\theta_{i}$ be the generator of $H^{2 i-1}\left(S^{2 i-1}\right)$ for each $i$ and $x_{1}, x_{3}, \ldots, x_{2 n-1}$ be generators of the ring $H^{*}(U(n))$ in each dimension. Then by Lemma 1.5 we have $u_{i}^{*} \theta=k_{i} x_{2 i-1}$. It is easy to see that $u=u_{1} \times u_{2} \times \cdots \times u_{n}: U(n) \rightarrow$ $S^{1} \times S^{3} \times \cdots \times S^{2 n-1}$ is the map we want. Q.E.D.

Now Theorem 1.2 just follows from Lemma 1.2, Lemma 1.3 and Lemma 1.7 where we use the Chern map again.

Corollary. Let $X$ be a compact metrizable space. For any $\tau \in H^{2 n-1}(X)$, there exist an integer $m \neq 0$ and $f: X \rightarrow S^{2 n-1}$ such that $m \tau=f^{*} \theta$, where $\theta$ is the generator of $H^{2 n-1}\left(S^{2 n-1}\right)$.

Proof. This is a direct consequence of Theorem 1.2 using the Chern map. Q.E.D.

Remark 1. Lemma 1.6 and Lemma 1.7 state that there exist maps from $U(n)$ to spheres which realize the multipliers of the generators of the cohomology ring of $U(n)$. It would be nice to find the maps explicitly, but the author does not know any direct way to do it.

Remark 2. The following result can be concluded from [19 and 7]: If $X$ is a finite CW complex and $\operatorname{dim} X<2 k-1$, then for any $\tau \in H^{k}(X)$, there exist a map $f: X \rightarrow S^{k}$ and an integer $m \neq 0$ such that $m \tau=f^{*} \theta$. Our Corollary is a similar result which has no restriction on the dimension of $X$. And we should point out the following facts: (1) Theorem 1.2 and the Corollary are not true for $K^{0}(X)$ and $H^{2 n}(X)$ (we give a counterexample below). (2) In Theorem 1.2 and the Corollary, it is essential to have a multiplier of $\tau$. Generally, we cannot find $f_{i}$ with $\tau=\sum_{i=1}^{n} f_{i}^{*} \theta_{i}$ or $f$ with $\tau=f^{*} \theta$.

Remark 3. By [16, top line on p. 165], $U(n)$ and $S^{1} \times S^{3} \times \cdots \times S^{2 n-1}$ have the same rational homotopy type. But this is not enough to conclude the existence of the map $u$ in Lemma 1.7.

We give the following example which shows that Theorem 1.2 and its Corollary are not true for the even case. 
Example. Let $X$ be the complex projective plane $\mathbb{C} P^{2}$. It is well known that $H^{2}(X) \cong H^{4}(X) \cong \mathbb{Z}$ and furthermore, if $\theta_{1}$ and $\theta_{2}$ are the generators of $H^{2}(X)$ and $H^{4}(X)$ respectively, then $\theta_{1}^{2}=\theta_{2}$. If $f: X \rightarrow S^{2}$ is a continuous map and $\theta$ is the generator of $H^{2}\left(S^{2}\right)$, then

$$
\left(f^{*}(\theta)\right)^{2}=f^{*}\left(\theta^{2}\right)=f^{*}(0)=0 .
$$

Hence $f^{*}(\theta)=0$. So neither $\theta_{1}$ nor multipliers of $\theta_{1}$ can be expressed by pullback of $\theta$.

The author would like to thank the referee who kindly points out the above example which is simpler than the one in the old version of the paper.

We conclude this section with the following theorem which is a special case of the theorem on p. 210 on line 7 of [21].

Theorem 1.3. If $(X, Y)$ is a finite $C W$ complex pair and $\tau \in H_{k}(X, Y)$, then there exist a smooth compact oriented $k$-manifold $M$ with boundary $\partial M$ and a map $f: M \rightarrow X$ with $f(\partial M) \subset Y$ such that $m \tau=f_{*} \theta$ for some integer $m \neq 0$ and $\theta \in H_{k}(M, \partial M)$. In particular, if $Y$ is empty, then $M$ can be chosen as a smooth compact oriented manifold without boundary.

\section{SMOOTH EXTENSIONS FOR FINITE CW COMPLEXES}

In this section, we study $C_{p}$-smoothness of extensions for finite CW complexes and $\mathrm{CW}$ complex pairs. The notion of $C_{p}$-smoothness was introduced by $R$. Douglas and studied in [17, 18,11 and 12]. In this aspect, the two most important results are the following:

(1) $C_{1}$-smooth elements of $\operatorname{Ext}(X)$ come from the 1-skeleton of $X$ modulo torsion $[17,11]$, when $X$ is a simplicial complex.

(2) $C_{n-1}$-smooth elements of $\operatorname{Ext}\left(S^{2 n-1}\right)$ are trivial [12].

The natural problem is to characterize the $C_{p}$-smooth extensions for a general space $X$ (e.g. CW complex). In this section, we first generalize the definition of $C_{p}$-smoothness (Definition 0.1 in the Introduction) to a finite $\mathrm{CW}$ complex. And we obtain necessary and sufficient conditions for an extension of a finite $\mathrm{CW}$ complex to be $C_{p}$-smooth modulo torsion. In particular, the above results (1) and (2) are direct consequences of our theorem. We also prove a similar result for a relative extension of a $\mathrm{CW}$ complex pair. Our results answer several open questions in [11 and 12]. Finally, we apply our theorem to operator theory and study an essentially normal $n$-tuple of operators $\left(T_{1}, T_{2}, \ldots, T_{n}\right)$ with the commutators $\left[T_{i}, T_{j}^{*}\right]$ and $\left[T_{i}, T_{j}\right](1 \leq i, j \leq n)$ in $C_{p}$-class.

Let $H, L(H), K(H), Q(H)$ and $\pi: L(H) \rightarrow Q(H)$ be as in the Introduction. Recall that if $T$ is a compact operator on $H$, then there exists a complete orthonormal basis $\left\{\psi_{n}\right\}_{n=1}^{\infty}$ of $H$ such that $T^{*} T \psi_{n}=\lambda_{n}^{2} \psi_{n}$ where $\left\{\lambda_{n}\right\}_{n=1}^{+\infty}$ is a sequence of nonnegative real numbers with $\lambda_{n} \rightarrow 0$. We say $T$ is in the Schatten-von Neuman $p$-class (denoted by $T \in C_{p}$ ), if $T \in K(H)$ and $\sum_{n=1}^{\infty} \lambda_{n}^{p}<+\infty$. It is obvious that $C_{p} \subset C_{q}$ when $p<q$. Therefore, in Definition 0.1 in the Introduction, if $T$ is $C_{p}$-smooth, then $T$ is $C_{q}$-smooth whenever $q>p$.

Theorem 1.1 in $\S 1$ is used to give the following definition of $C_{p}$-smooth for a finite $\mathrm{CW}$ complex. 
Definition 2.1. Let $X$ be a finite $\mathrm{CW}$ complex, and $M$ and $f$ be as in Theorem 1.1. Then $\tau \in \operatorname{Ext}(X)$ is $C_{p}$-smooth if $f_{*} \tau \in \operatorname{Ext}(M)$ is $C_{p}$-smooth (see Definition 0.1).

Theorem 2.1. The definition of $C_{p}$-smoothness does not depend on the choice of $M$ or on the maps $f$ and $g$ in Theorem 1.1 .

To prove Theorem 2.1, we need the following lemma:

Lemma 2.1. Let $M$ and $N$ be compact smooth manifolds (perhaps with boundary), and let $u$ be a continuous map between $M$ and $N$. If $\tau \in \operatorname{Ext}(M)$ is $C_{p}$-smooth, then $u_{*} \tau \in \operatorname{Ext}(N)$ is $C_{p}$-smooth.

Proof. By Proposition 17.8 on p. 213 of [5], there exists a smooth map $v$ between $M$ and $N$ such that $u$ is homotopy equivalent to $v$. So we can assume $u$ is a smooth map.

Let $\tau: C(M) \rightarrow Q(H)$ be a $C_{p}$-smooth extension. Then by Definition 0.1 there exists a positive linear $*$ map $\rho: C^{\infty}(M) \rightarrow L(H)$ such that $\rho \circ \pi=\tau$ and $\rho(f g)-\rho(f) \rho(g) \in C_{p}$ for all $f, g \in C^{\infty}(M)$.

Let us describe $u_{*} \tau \in \operatorname{Ext}(N)$. First we define $\tau_{1}: C(N) \rightarrow Q(H)$ as the following:

$$
\tau_{1}(f)=\tau(f \circ u) \text { for } f \in C(N) .
$$

Then $\tau_{1}$ is a $*$ homomorphism. But in general, $\tau_{1}$ is not faithful. So we cannot say $u_{*} \tau$ is determined by $\tau_{1}$. However, if $\tau_{2}: C(N) \rightarrow Q(H)$ is a faithful * homomorphism which can be lifted to a * homomorphism $\rho_{2}: C(N) \rightarrow$ $L(H)$ (this means that $\tau_{2}$ is trivial in $\left.\operatorname{Ext}(N)\right)$, then $u_{*} \tau$ is determined by $\tau_{1} \oplus \tau_{2}: C(N) \rightarrow Q(H \oplus H)$. Let $\rho_{1}: C^{\infty}(N) \rightarrow L(H)$ be a positive linear map determined by $\rho_{1}(f)=\rho(f \circ u)$ for $f \in C^{\infty}(N)$. It is easy to verify that $\rho_{1} \oplus\left(\left.\rho_{2}\right|_{C^{\infty}(N)}\right): C^{\infty}(N) \rightarrow L(H \oplus H)$ satisfies the condition in Definition 0.1 which serves as the lifting for $\tau_{1} \oplus \tau_{2}$. Therefore $u_{*} \tau$ is $C_{p}$-smooth. Q.E.D.

Proof of Theorem 2.1. Let $M_{1}$ and $f_{1}, g_{1}$ be another choice of space and maps which satisfies Theorem 1.1. Because $g \circ f$ is homotopic to id $\left.\right|_{X}, f_{1}$ is homotopic to $f_{1} \circ g \circ f$. Therefore

$$
f_{1 *} \tau=\left(f_{1} \circ g\right)_{*}\left(f_{*} \tau\right) \text {. }
$$

By Lemma 2.1, if $f_{*} \tau$ is $C_{p}$-smooth, then $f_{1 *} \tau$ is $C_{p}$-smooth. The proof of the converse is exactly the same. Q.E.D.

The following two corollaries are direct consequences of Lemma 2.1 and its proof.

Corollary 1. In Definition 0.1 , the notion of $C_{p}$-smoothness does not depend on the particular smooth structure associated to the manifold $M$.

Proof. Take $f$ to be the identity map on $M$ in Lemma 2.1. Q.E.D.

Corollary 2. Let $f: X \rightarrow Y$ be a continuous map. Then $f_{*}$ takes the $C_{p}$-smooth elements of $\operatorname{Ext}(X)$ to $C_{p}$-smooth elements of $\operatorname{Ext}(Y)$.

Corollary 1 above answers the question on p. 68 of [11].

Now let $M$ be a smooth compact oriented manifold without boundary. In [2], Baum and Douglas construct an element in $\operatorname{Ext}(M)$ for each selfadjoint elliptic pseudo-differential operator on $M$. This construction and Theorem 2 in [2] can be used to prove the following theorem. However, we believe that this theorem is well known to experts, so we omit the proof. 
Theorem 2.2. If $M$ is an n-dimensional oriented compact smooth manifold without boundary, then all the elements of $\operatorname{Ext}(M)$ are $C_{p}$-smooth whenever $p>\frac{n}{2}$.

Now we are going to prove our main results in this section.

Theorem 2.3. Let $X$ be a finite $C W$ complex, $\tau \in \operatorname{Ext}(X)=K_{1}(X)$ and ch: $K_{1}(X) \otimes \mathbb{Q} \rightarrow H_{\text {odd }}(X, \mathbb{Q})$ be the Chern map, where $H_{\text {odd }}(X, \mathbb{Q})$ denotes the direct sum of all the ordinary homology groups of odd dimension with rational coefficients. Then there exists an integer $m \neq 0$ such that $m \tau$ is $C_{n}$-smooth if and only if ch $\tau \in \bigoplus_{k=1}^{n} H_{2 k-1}(X, \mathbb{Q})$.

Proof. If $\operatorname{ch} \tau \in \bigoplus_{k=1}^{n} H_{2 k-1}(X, \mathbb{Q})$, then $\operatorname{ch} \tau=\theta_{1}+\theta_{3}+\cdots+\theta_{2 n-1}$, where $\theta_{2 i-1} \in H_{2 i-1}(X, \mathbb{Q})$. Without loss of generality, we can assume ch $\tau=\theta \in$ $H_{2 n-1}(X, \mathbb{Q})$. By Theorem 1.3 in $\S 1$, there exist a compact oriented smooth manifold $M$ of dimension $2 n-1$ and a map $f: M \rightarrow X$ such that $\theta=f_{*} \tilde{\theta}$ where $\tilde{\theta} \in H_{2 n-1}(M, \mathbb{Q})$. (Please note that we use rational coefficients here, so we do not need to multiply $\theta$ by an integer.) By surjectivity of the Chern map between $K_{1}(M) \otimes \mathbb{Q}$ and $H_{\text {odd }}(M, \mathbb{Q})$, there exist a $\tilde{\tau} \in \operatorname{Ext}(M)=K(M)$ and a rational number $\frac{p}{q}$ with $\operatorname{ch}\left(\frac{p}{q} \tilde{\tau}\right)=\theta$. Therefore, $\operatorname{ch} \tau=f_{*} \operatorname{ch}\left(\frac{p}{q} \tilde{\tau}\right)=\operatorname{ch}\left(f_{*}\left(\frac{p}{q} \tilde{\tau}\right)\right)$. Using the injectivity of the Chern map,

$$
\tau=\frac{p}{q}\left(f_{*} \tilde{\tau}\right) \text { in } K_{1}(X) \otimes \mathbb{Q} .
$$

Hence, there exists an integer $m_{1}$ with

$$
m_{1} \tau=m_{1} p\left(f_{*} \tilde{\tau}\right) / q \text { in } K_{1}(X) .
$$

Therefore,

$$
m_{1} q \tau=m_{1} p\left(f_{*} \tilde{\tau}\right)=f_{*}\left(m_{1} p \tilde{\tau}\right) .
$$

Let $m=m_{1} q$. From Theorem 2.2 and Corollary 2 of Theorem 2.1, we know that $m \tau$ is $C_{n}$-smooth. This completes the proof of the "if" part.

Suppose that there exists an integer $m \neq 0$ with $m \tau$ being $C_{n}$-smooth. We are going to prove $\operatorname{ch} \tau \in \bigoplus_{k=1}^{n} H_{2 k-1}(X, \mathbb{Q})$. If not, there exists a $\theta \in$ $H^{2 i-1}(X, \mathbb{Q})$ with $i>n$, such that the pairing $\langle\operatorname{ch} \tau, \theta\rangle \neq 0$. By the Corollary of Theorem 1.2, there exist a map $f: X \rightarrow S^{2 i-1}$ and $\tilde{\theta} \in H^{2 i-1}\left(S^{2 i-1}\right)$ such that $\theta=f^{*} \frac{p}{q} \tilde{\theta}$ where $\frac{p}{q}$ is a rational number. By our assumption, $m \tau$ is $C_{n^{-}}$ smooth. Therefore $m f_{*} \tau=f_{*} m \tau$ is a $C_{n}$-smooth element in $\operatorname{Ext}\left(S^{2 i-1}\right)$. By Proposition 3 in [12], $m f_{*} \tau$ is the trivial element in $\operatorname{Ext}\left(S^{2 i-1}\right)=\mathbb{Z}$. Therefore $f_{*} \tau=0$. Hence

$$
\begin{aligned}
0 & =\left\langle\operatorname{ch} f_{*} \tau, \frac{p}{q} \tilde{\theta}\right\rangle=\left\langle f_{*} \operatorname{ch} \tau, \frac{p}{q} \tilde{\theta}\right\rangle \\
& =\left\langle\operatorname{ch} \tau, f^{*}\left(\frac{p}{q} \tilde{\theta}\right)\right\rangle=\langle\operatorname{ch} \tau, \theta\rangle \neq 0 .
\end{aligned}
$$

This contradiction completes the proof of the "only if" part of Theorem 2.3. Q.E.D.

The following theorem is almost equivalent to the above results but is perhaps more useful in practice.

Theorem 2.4. Let $X$ be a finite $C W$ complex, $X^{k}$ denote the $k$-skeleton of $X$ and $\tau \in \operatorname{Ext}(X)$. Then there exists an integer $m_{1} \neq 0$ such that $m_{1} \tau$ 
is $C_{n}$-smooth if and only if there exists an integer $m_{2} \neq 0$ such that $m_{2} \tau \in$ $i_{*}\left(\operatorname{Ext} X^{2 n-1}\right)$, where $i_{*}: \operatorname{Ext}\left(X^{2 n-1}\right) \rightarrow \operatorname{Ext}(X)$ is induced by the inclusion map $i: X^{2 n-1} \rightarrow X$.

Proof. If there exists an integer $m_{1} \neq 0$ with $m_{1} \tau$ being $C_{n}$-smooth, then by Theorem 2.3, ch $\tau \in \bigoplus_{k=1}^{n} H_{2 k-1}(X, \mathbb{Q})$. Please note the following wellknown fact from homology theory, $i_{*}: H_{k}\left(X^{n}\right) \rightarrow H_{k}(X)$ is a bijection when $k<n$ and a surjection when $k=n$. It is routine to prove that there exists an integer $m_{2} \neq 0$ with $m_{2} \tau \in i_{*}\left(X^{2 n-1}\right)$. The "if" part follows from Theorem 2.2 , Theorem 2.3 and the bijectivity of the Chern map from $K_{1}(X) \otimes \mathbb{Q}$ to $H_{\text {odd }}(X) \otimes \mathbb{Q}$. Q.E.D.

Corollary 1. If $X$ is a $(2 n-1)$-connected finite $C W$ complex, then all the $C_{n^{-}}$ smooth elements of $\operatorname{Ext}(X)$ are torsion elements.

Proof. As in $\S 1$, we know $X$ is homotopic to a CW complex with its $2 n-1$ skeleton being a single point. According to Corollary 2 of Theorem 2.1, we can assume that the $2 n-1$ skeleton of $X$ is one point. Thus this corollary follows from Theorem 2.4. Q.E.D.

Corollary 2. Let $X$ be a $(2 n-1)$-connected finite $C W$ complex and $\operatorname{dim} X \leq$ $2 n+1$. Then all the $C_{n}$-smooth elements of $\operatorname{Ext}(X)$ are trivial.

Proof. Let $\tau \in \operatorname{Ext}(X)$ be $C_{n}$-smooth. According to Corollary 1, there exists an integer $m \neq 0$ with $m \tau=0$. Consider the relative homology exact sequence of $\left(X, X^{2 n}\right)$, where $X^{2 n}$ is the $2 n$-skeleton of $X$ (see p. 37 of [10] for the sequence).

$$
\operatorname{Ext}\left(X^{2 n}\right) \stackrel{i_{*}}{\rightarrow} \operatorname{Ext}(X) \stackrel{p_{*}}{\rightarrow} \operatorname{Ext}\left(X / X^{2 n}\right) .
$$

Since $\operatorname{dim} X \leq 2 n+1, X / X^{2 n}$ is the space of the bouquet $S^{2 n+1} \vee S^{2 n+1} \vee \cdots \vee$ $S^{2 n+1}$ of several $2 n+1$ dimensional spheres. This implies that $\operatorname{Ext}\left(X / X^{2 n}\right)$ is torsion free. Therefore $p_{*} \tau=0$. So $\tau=i_{*} \theta$ for $\theta \in \operatorname{Ext}\left(X^{2 n}\right)$. But $X$ is $(2 n-1)$-connected, so we can assume that $X^{2 n-1}$ is one point. Hence $X^{2 n}$ is the bouquet of $2 n$-dimensional spheres. Therefore $K^{1}\left(X^{2 n}\right)=0$. This completes the proof of Corollary 2. Q.E.D.

The main theorem of [12] (see Proposition 3 in that paper) is the special case of our Corollary 1 when we take $X$ to be $S^{2 n+1}$. And the two main theorems of [11] (see p. 65 and p. 66) are the special cases of our Corollary 1 and Corollary 2 respectively taking $n=1$. But we should point out that Proposition 3 in [12] is used in proving our main results and that the original proof of the theorem in [11] inspired our proof of Corollary 2.

Now, we will briefly discuss the $C_{p}$-smoothness for a relative extension of a finite $\mathrm{CW}$ complex pair.

Definition 2.2. Let $(X, Y)$ be a relative finite $\mathrm{CW}$ complex pair. Then $\tau \in$ $\operatorname{Ext}(X, Y)$ is said to be $C_{p}$-smooth if the image of $\tau$ under the canonical isomorphism from $\operatorname{Ext}(X, Y)$ to $\operatorname{Ext}(X / Y)$ is $C_{p}$-smooth.

One can prove the following theorem.

Theorem 2.5. Let $\tau \in \operatorname{Ext}(X, Y)$. Then the following are equivalent:

1. There exists an integer $m \neq 0$ such that $m \tau$ is $C_{n}$-smooth.

2. $\operatorname{ch} \tau \in \bigoplus_{k=1}^{n} H_{2 k-1}(X, Y, \mathbb{Q})$, where $H_{i}(X, Y, \mathbb{Q})$ denotes the relative homology group of the $C W$ complex pair with rational coefficients. 
Before we end this section we give an application of our theorem to operator theory.

Let $X$ be a finite simplicial complex embedded in $R^{n}$. We say that $X$ is smoothly embedded in $R^{n}$ if we can find a closed neighborhood $U$ with smooth boundary, such that $X$ is a retract of $U$ and the retraction $r: U \rightarrow X$ is homotopic to a map $f: U \rightarrow X$ with $i \circ f: U \rightarrow X \rightarrow U$ being smooth. We can prove that any 1-dimensional simplicial complex can be smoothly embedded in $R^{n}$. And while I believe that this is true for any dimension, I have been unable to prove it.

We give the following theorem as an application of our results in this section.

Theorem 2.6. Let $\left(T_{1}, T_{2}, \ldots, T_{n}\right)$ be an essentially normal $n$-tuple of operators and $X \subset \mathbb{C}^{n}$ be the essential spectrum of the $n$-tuple. Then the following statements are true:

(1) If $X$ is a closed m-dimensional smooth oriented manifold embedded in $\mathbb{C}^{n}$, then for any $p>m / 2$, there exists an n-tuple of compact operators $\left(K_{1}, K_{2}, \ldots, K_{n}\right)$ with $\left[T_{i}+K_{i}, T_{j}+K_{j}\right] \in C_{p}$ and $\left[T_{i}+K_{i}, T_{j}^{*}+K_{j}^{*}\right] \in C_{p}$.

(2) If $X$ is a m-dimensional simplicial complex smoothly embedded in $\mathbb{C}^{n}$, then for some $n$-tuple

$$
\begin{aligned}
& \left(S_{1}, S_{2}, \ldots, S_{n}\right) \\
& \quad=(\underbrace{T_{1} \oplus T_{1} \oplus \cdots \oplus T_{1}}_{k \text {-copies }}, \underbrace{T_{2} \oplus T_{2} \oplus \cdots \oplus T_{2}}_{k \text {-copies }}, \ldots, \underbrace{T_{n} \oplus T_{n} \oplus \cdots \oplus T_{n}}_{k \text {-copies }})
\end{aligned}
$$

( $k$ is an integer), there exists an $n$-tuple of compact operators $\left(K_{1}, K_{2}, \ldots, K_{n}\right)$ acting on

$$
\underbrace{H \oplus H \oplus \cdots \oplus H}_{k \text {-copies }}
$$

such that $\left[S_{i}+K_{i}, S_{j}+K_{j}\right] \in C_{[m / 2]+1}$ and $\left[S_{i}+K_{i}, S_{j}^{*}+K_{j}^{*}\right] \in C_{[m / 2]+1}$.

Proof. (1) follows from Theorem 2.2 and (2) follows from Theorem 2.4 and the Corollary 2 of Theorem 2.1. Q.E.D.

If we take $X=S^{2 n-1}$, then we answer the question on p. 109 of [12]. In particular, we have the following fact: If $\left(T_{z_{1}}, T_{z_{2}}, \ldots, T_{z_{n}}\right)$ is the $n$ tuple of Toeplitz operators on $H^{2}\left(\partial B_{n}\right)$, then there exist $n$ compact operators $\left(K_{1}, K_{2}, \ldots, K_{n}\right)$ such that $\left[T_{z_{i}}+K_{i}, T_{z_{j}}+K_{j}\right] \in C_{p}$ and $\left[T_{z_{i}}+K_{i}, T_{z_{j}}^{*}+K_{j}^{*}\right] \epsilon$ $C_{p}$ when $p>n-\frac{1}{2}$. There does not seem to be any direct proof of this fact.

\section{Summable Fredholm modules of $C^{\infty}(M)$ FOR A COMPACT SMOOTH MANIFOLD $M$}

Corresponding to $K_{1}(X)=\operatorname{Ext}(X)$ [6, 10], in the even case, Kasparov [20] proved that each element of $K_{0}(X)$ can be realized as a Fredholm module of $C(X)$ and therefore $K_{0}(X)=K K(C(X), \mathbb{C})$. We refer to [4] for the general theory of $K$-homology and $K K$-groups.

Let $M$ be a compact smooth manifold, and $C^{\infty}(M)$ denote the algebra of smooth functions on $M$. The notion of a $p$-summable Fredholm module of $C^{\infty}(M)$, which can be thought of as an element in $K_{0}(M)=K K(C(X), \mathbb{C})$, is the even analogy of $C_{p}$-smooth extension and it was introduced by Connes [8]. 
In this section, we study the $p$-summable Fredholm modules of $C^{\infty}(M)$ and their Chern characters in $H_{\lambda}^{*}\left(C^{\infty}(M)\right)$, where $H_{\lambda}^{*}\left(C^{\infty}(M)\right)$ is the cyclic cohomology of $C^{\infty}(M)$. Furthermore, we also study the case of a compact manifold with boundary.

First we briefly recall some definitions and basic results. Let $X$ be a compact metrizable space, and $\Gamma(X)$ denote the collection of triples $(H, \sigma, F)$ which are called the Fredholm modules of $C(X)$, where,

(1) $H=H_{0} \oplus H_{1}$ is a $\mathbb{Z}_{2}$ graded Hilbert space with a grading operator $\mathscr{E}$, $\mathscr{E} \xi=(-1)^{\operatorname{deg} \xi} \xi$ for all $\xi \in H_{0}$ or $\xi \in H_{1}$;

(2) $\sigma=\sigma_{0} \oplus \sigma_{1}$, and $\sigma_{i}: C(X) \rightarrow L\left(H_{i}\right)$ is a continuous $*$ homomorphism;

(3) $F \in L(H), F^{2}=I, F \mathscr{E}=-\mathscr{E} F$, and for any $f \in C(X)$, one has

$$
F \sigma(f)-\sigma(f) F \in K(H) \text {. }
$$

$K_{0}(X)$ is defined to be $\Gamma(X)$ modulo certain equivalence relations (for details see $\S 2$ of [3] or Chapter 5 of [10]).

If $M$ is a compact smooth manifold, then a $p$-summable Fredholm module of $C^{\infty}(M)$ is an element of $\Gamma(M)$ which satisfies the following stronger condition:

(3') $F \in L(H), F^{2}=I, F \mathscr{E}=-\mathscr{E} F$, and for any $f \in C^{\infty}(M)$, one has

$$
F \sigma(f)-\sigma(f) F \in C_{p} \text {. }
$$

In [8], Connes defined the cyclic cohomology $H_{\lambda}^{n}(\mathscr{A})$ of an algebra $\mathscr{A}$ over $\mathbb{C}$ as follows. Let $C_{\lambda}^{n}(\mathscr{A})$ be the set of $n+1$ linear functions on $\mathscr{A}$ which satisfy

$$
\begin{aligned}
& \tau\left(a^{1}, a^{2}, \ldots, a^{n}, a^{0}\right) \\
& \quad=(-1)^{n} \tau\left(a^{0}, a^{1}, a^{2}, \ldots, a^{n}\right), \quad \forall a^{0}, a^{1}, a^{2}, \ldots, a^{n} \in \mathscr{A} .
\end{aligned}
$$

Define $b: C_{\lambda}^{n}(\mathscr{A}) \rightarrow C_{\lambda}^{n+1}(\mathscr{A})$ by

$$
\begin{aligned}
(b \tau) & \left(a^{0}, a^{1}, a^{2}, \ldots, a^{n+1}\right)=\tau\left(a^{0} a^{1}, a^{2}, \ldots, a^{n+1}\right) \\
& +\sum_{i=1}^{n}(-1)^{i} \tau\left(a^{0}, \ldots, a^{i} a^{i+1}, \ldots, a^{n+1}\right) \\
& +(-1)^{n+1} \tau\left(a^{n+1} a^{0}, \ldots, a^{n}\right) .
\end{aligned}
$$

One can verify $b^{2}=0 . H_{\lambda}^{n}(\mathscr{A})$ is defined to be

$$
\frac{\operatorname{Ker}\left\{b: C_{\lambda}^{n}(\mathscr{A}) \rightarrow C_{\lambda}^{n+1}(\mathscr{A})\right\}}{\operatorname{Im}\left\{b: C_{\lambda}^{n-1}(\mathscr{A}) \rightarrow C_{\lambda}^{n}(\mathscr{A})\right\}} .
$$

Connes defined a useful map $S: H_{\lambda}^{n}(\mathscr{A}) \rightarrow H_{\lambda}^{n+2}(\mathscr{A})$, and defined $H^{\text {even }}(\mathscr{A})$ to be the inductive limit of the groups $H_{\lambda}^{2 n}(\mathscr{A})$ under the map $S: H_{\lambda}^{2 n}(\mathscr{A}) \rightarrow$ $H_{\lambda}^{2 n+2}(\mathscr{A})$, or equivalently, the quotient of $\bigoplus_{n=1}^{\infty} H_{\lambda}^{2 n}(\mathscr{A})$ by the equivalence relation $\varphi \sim S \varphi . H^{\text {odd }}(\mathscr{A})$ is defined in the same way.

In $\S 2$ of [8], Connes also constructed the graded Chern map

$\mathrm{ch}^{*}:\{(n+1)$-summable Fredholm modules $\} \rightarrow H_{\lambda}^{n}\left(C^{\infty}(M)\right)$,

where $n$ is an even integer. And he proved that

$\mathrm{ch}^{*}:\{$ finite summable Fredholm modules $\} \rightarrow H^{\text {even }}\left(C^{\infty}(M)\right)$

is a surjection up to complex multipliers. Our main result in this section says that the Chern map is a graded surjection. 
Theorem 3.1. Let $M$ be an $n$-dimensional compact oriented smooth manifold ( $n$ even). Then all the elements of $K_{0}(M)$ can be realized as $(n+1)$-summable Fredholm modules.

Proof. In $\S 5$ of [2], Baum-Douglas proved that each element $\tau$ in $K_{0}(M)$ can be represented by a first order elliptic pseudo-differential operator. Then by $\S 6$ of [8], we know that $\tau$ can be realized as an $(n+1)$-summable Fredholm module. This completes the proof. Q.E.D.

Before proving our main result, we give the following two lemmas.

Lemma 3.1. If $X$ is a finite $C W$ complex and $\tau \in H_{k}(X, \mathbb{C})$, then there exist compact oriented smooth $k$-manifolds $M_{i}(i=1,2, \ldots, n)$ without boundary and maps $f_{i}: M_{i} \rightarrow X$ such that $\tau=\sum_{i=1}^{n} f_{i *} \theta_{i}$ for some $\theta_{i} \in H_{k}\left(M_{i}, \mathbb{C}\right)$, where $H_{k}(X, \mathbb{C})$ denote the homology of $X$ with complex coefficients.

This lemma is another version of Theorem 1.3. We omit the proof here.

Please note that one should use several manifolds rather than a single manifold (in Theorem 1.3) because of the complex coefficients.

Lemma 3.2. If $M, N$ are compact smooth manifolds and $f: M \rightarrow N$ is a continuous map, then $f_{*}$ maps p-summable elements of $K_{0}(M)$ to p-summable elements of $K_{0}(N)$.

The proof of this lemma is exactly the same as that of Lemma 2.1.

Theorem 3.2. If $M$ is a compact smooth manifold without boundary and $\varphi \in$ $H_{\lambda}^{k}\left(C^{\infty}(M)\right)$ ( $k$ even), then there exist $(k+1)$-summable Fredholm modules $\tau_{i}(i=1,2, \ldots, n)$ and complex numbers $\alpha_{i}(i=1,2, \ldots, n)$ such that $\sum_{i=1}^{n} \alpha_{i} \operatorname{ch}^{*} \tau_{i} \sim \varphi$ in $H^{*}\left(C^{\infty}(M)\right)$, where $\mathrm{ch}^{*}$ is Connes' Chern map.

Proof. Let $\varphi \in H_{\lambda}^{k}\left(C^{\infty}(M)\right)$. According to (1) and (2) of Theorem 46 in [8], we know that $\varphi$ corresponds to $\varphi_{k}+\varphi_{k-2}+\cdots+\varphi_{0}$ under a map $h: H^{\text {even }}\left(C^{\infty}(M)\right)$ $\rightarrow \bigoplus_{j} H_{2 j}(M, \mathbb{C})$, where $\varphi_{k-j} \in H_{k-j}(M, \mathbb{C})$. As pointed out by Connes in [8], the composition of the following two maps

$$
K_{0}(M) \otimes \mathbb{C} \stackrel{\mathrm{ch}^{*}}{\longrightarrow} H^{\text {even }}\left(C^{\infty}(M)\right) \stackrel{h}{\rightarrow} \bigoplus_{j} H_{2 j}(M, \mathbb{C})
$$

is the usual Chern map from $K_{0}(M) \otimes \mathbb{C}$ to $\bigoplus_{j} H_{2 j}(M, \mathbb{C})$ (denoted by ch), where $\mathrm{ch}^{*}$ is induced by Connes' Chern map $\mathrm{ch}^{*}: K_{0}(M) \rightarrow H^{\text {even }}\left(C^{\infty}(M)\right)$. Therefore the composition of the following sequence of maps

$$
H^{\text {even }}\left(C^{\infty}(M)\right) \stackrel{h}{\rightarrow} \bigoplus_{j} H_{2 j}(M, \mathbb{C}) \stackrel{\mathrm{ch}^{-1}}{\longrightarrow} K_{0}(M, \mathbb{C}) \stackrel{\mathrm{ch}^{*}}{\longrightarrow} H^{\text {even }}\left(C^{\infty}(M)\right)
$$

is the identity map. From the above fact, we need only to prove that for each $\varphi_{k-j}$, there exist $k+1$-summable Fredholm modules $\tau_{i} \in K_{0}(M) \quad(i=$ $1,2, \ldots, n)$ and $\alpha_{i} \in \mathbb{C}(i=1,2, \ldots, n)$ such that $\operatorname{ch}^{-1} \varphi_{k-j}=\sum_{i=1}^{n} \alpha_{i} \tau_{i}$ as elements of $K_{0}(M) \otimes \mathbb{C}$.

Without loss of generality, we just need to prove this for $\varphi_{k}$.

Using Lemma 3.1, we have $\varphi_{k}=\sum_{i=1}^{n} f_{i *} \theta_{i}$, where $\theta_{i} \in H_{k}\left(M_{i}, \mathbb{C}\right)$ and $M_{i}$ is a connected compact oriented smooth $k$-manifold. Since $H_{k}\left(M_{i}\right) \otimes \mathbb{C} \cong$ $H_{k}\left(M_{i}, \mathbb{C}\right)=\mathbb{C}$ as well as $H_{k}\left(M_{i}, \mathbb{Z}\right)=\mathbb{Z}$, there exists a $\beta_{i} \in \mathbb{C} \backslash\{0\}$ such that $\beta_{i} \theta_{i}$ corresponds to an integer under the isomorphism between $H_{i}(M, \mathbb{C})$ 
and $\mathbb{C}$. This means that $\beta_{i} \theta_{i}$ can be expressed as an element of $H_{k}\left(M_{i}, \mathbb{Z}\right)$. Therefore $\operatorname{ch}^{-1} \beta_{i} \theta_{i} \in K_{0}(M) \otimes \mathbb{C}$ can be chosen as an element in $K_{0}\left(M_{i}\right)$. Hence $\operatorname{ch}^{-1} \beta_{i} \theta_{i}$ can be represented by a $(k+1)$-summable Fredholm module according to Theorem 3.1. Lemma 3.2 says that $f_{i *} \operatorname{ch}^{-1} \beta_{i} \theta_{i} \in K_{0}(M)$ can be represented by a $k+1$-summable Fredholm module. But

$$
\operatorname{ch}^{-1} \varphi_{k}=\sum_{i=1}^{n} \frac{1}{\beta_{i}} \tau_{i}
$$

as the element in $K_{0}(M) \otimes \mathbb{C}$, where $\tau_{i}=f_{i *} \mathrm{ch}^{-1} \beta_{i} \theta_{i}$. This completes the proof. Q.E.D.

\section{ACKNOWLEDGMENTS}

I would like to express my most sincere gratitude to my advisor, Professor Ronald G. Douglas who suggested the topic of this paper and shared with me his fruitful ideas and penetrating insights. Special thanks go to Professor Nicholae Teleman and Dr. John Spielberg, Guoliang Yu and Xianzhe Dai for several helpful discussion.

\section{REFERENCES}

1. M. Atiyah, Global theory of elliptic operators, Proc. Internat. Conf. on Functional Analysis and Related Topics, Univ. of Tokyo Press, Tokyo, 1970.

2. P. Baum and R. Douglas, Toeplitz operators and Poincare duality, Toeplitz Memorial Conference, Tel Aviv, 1981.

3. _ـ Relative K-homology and $C^{*}$-algebra, manuscript.

4. B. Blackadar, K-theory for operator algebras, Math. Sci. Res. Inst. Publ. No. 5, SpringerVerlag, New York, 1986.

5. R. Bott and L. Tu, Differential forms in algebraic topology, Graduate Texts in Math. 82, Springer-Verlag, New York, 1982.

6. L. Brown, R. Douglas, and P. Fillmore, Extensions of $C^{*}$-algebra and K-homology, Ann. of Math. (2) 165 (1977), 265-324.

7. C. Chen, $A$ note on the classification of mapping of a $(2 n-2)$ dimensional complex into an n-sphere, Ann. of Math. 51 (1950), 238-240.

8. A. Connes, Non commutative differential geometry, Chapitres 1, 2, Publ. Math. Inst. Hautes Études Sci. 62 (1986), 257-360.

9. Cyclic cohomology and the tranverse fundamental class of a foliation, Preprint, IHES M18417, 1984.

10. R. Douglas, $C^{*}$-algebra extensions and $K$-homology, Ann. of Math. Stud. no. 95, Princeton Univ. Press, Princeton, N.J., 1980.

11. _ On the smoothness of elements of Ext. topics in modern operator theory, BirkhäuserVerlag, 1981, pp. 63-69.

12. R. Douglas and D. Voiculescu, On the smoothness of sphere extensions, J. Operator Theory 6 (1981), 103-111.

13. G. Gong, Smooth extensions for a finite $C W$ complex, Bull. Amer. Math. Soc. 22 (1990), 73-77.

14. - The relation between Ext group and index of Fredholm n-tuples, preprint.

15. B. Gray, Homotopy theory: an introduction to algebraic topology, Academic Press, New York, 1975.

16. P. Griffiths and J. Morgan, Rational homotopy theory and differential forms, Birkhäuser, Boston, Mass., 1981. 
17. J. Helton and R. Howe, Integral operators: commutators, traces index and homology, Proc. Conf. on Operator Theory, Lecture Notes in Math., vol. 345, Springer-Verlag, Berlin and New York, 1973, pp. 141-209.

18. _ Trace of commutators of integral operators, Acta Math. 135 (1975), 271-305.

19. S. T. Hu, Extension and classification of the mapping of a finite complex into a topological group or an n-sphere, Ann. of Math. 50 (1949), 158-173.

20. G. Kasparov, The operator $K$-functor and extensions of $C^{*}$-algebras, Izv. Akad. Nauk SSSR Ser. Math. 44 (1980), 571-636; English transl. in Math. USSR Izv.,16(, 1981), 513-572.

21. R. Stong, Note on corbordism theory, Math. Notes, Princeton Univ. Press, Princeton, N.J., 1968.

22. G. Whitehead, Elements of homotopy theory, Springer-Verlag, 1978.

Department of Mathematics, University of Toronto, Toronto, Canada M5S 1A1

Current address: Department of Mathematics, Jilin University, Changchun, China

E-mail address: guihua@math.utoronto.edu 